\title{
Der psychisch Kranke im Spiegel der Literatur: Das Beispiel Psychochirurgie
}

\author{
Dominik Groß und Gereon Schäfer
}

\section{Einleitung: Fragestellung und Vorannahmen}

Der Begriff „Psychochirurgie“ bezeichnet ablative und destruierende Eingriffe am morphologisch unauffälligen Gehirn mit dem Ziel der Beeinflussung psychischer Störungen. ${ }^{1}$ Es handelt sich hierbei um ein Verfahren, das von Medizinern entwickelt und besonders häufig im Zeitraum zwischen 1935 und 1960 eingesetzt worden ist. ${ }^{2}$ Im Fokus der Operateure standen bestimmte psychiatrische Krankheitsbilder wie Schizophrenie und bipolare affektive Störungen und deren unerwünschte soziale Folgen (z. B. mangelhafte Compliance im Anstaltsbetrieb bzw. „Unduldsamkeit“). Der Wegbereiter der Psychochirurgie, Egas Moniz, wurde 1949 - auf dem Höhepunkt des Verfahrens - mit dem Nobelpreis für Medizin geehrt. ${ }^{3}$ Doch diese Entscheidung des Preiskomitees und die damit ausgezeichnete Methode waren zu keinem Zeitpunkt unumstritten. ${ }^{4}$ Kritiker

1 Der vorliegende Aufsatz geht auf einen Vortrag zurück, den der Erstautor am 19.10.2006 im Rahmen der von Monika Fick ausgerichteten, DFG-finanzierten Tagung „Neuro-Imagination. Die phänomenale Welt im neurowissenschaftlichen und literarischen Diskurs" gehalten hat.

2 Antunes bezifferte die Zahl der aus psychiatrischer Indikation durchgeführten Eingriffe zwischen 1942 und 1954 in Großbritannien auf 11000; in den USA waren es im selben Zeitraum mindestens 18.000, vermutlich jedoch 50.000 Eingriffe: Antunes (2000), S. 241. Schipperges geht mit Blick auf die USA von 50.000 Eingriffen aus: Schipperges (1955), S. 2496. Ähnliche Zahlen nennt Koch (1976), S. 21.

3 Vgl. Fortner (2004); Fortner/Groß (2002); Groß (1999); Moniz (1949); News (1949).

4 Kritik an Moniz' Therapieverfahren übte beispielsweise der Board of Control: Im betreffenden Bericht wird der Fall eines Schizophrenen geschildert, dem es fünf Jahre nach der Operation „extrem gut“ ging und der keinerlei Rückfälle erlitten hatte, aber schließlich an einem Ösophaguskarzinom verstarb. Die durchgeführte Autopsie habe ergeben, dass die verbindenden Fasern zum Thalamus nicht durchschnitten worden seien und somit auch keine Degeneration des dorsomedialen Thalamuskerns eingetreten sei. Der Board of Control schlussfolgert daraus: „damages anywhere in the brain might have a benefit effect in some cases."Vgl. Board of Control (1947). 
verwiesen vor allem auf die Tatsache, dass bei den betroffenen Patienten keine kurative Wirkung erzielt werden konnte. Intendiert war vielmehr die Ruhigstellung der betreffenden Kranken (im Anstaltsbetrieb); sie beruhte auf einer postoperativen „Nivellierung“ der Persönlichkeit, die sich u.a. in einer Affektverflachung und einer Reduktion des Antriebs zeigte. Aus diesen Gründen geriet das Verfahren spätestens Ende der 1950er Jahre zunehmend in Misskredit, so dass die Zahl derartige Eingriffe weltweit drastisch abnahm. ${ }^{5}$

Unlängst entbrannte in Deutschland und Frankreich eine heftige Debatte über eine mögliche „Rückkehr der Psychochirurgie“. Anlass dieser aktuellen Diskussion sind Versuche einzelner Neurochirurgen, therapierefraktäre Zwangserkrankungen und Depressionen im Rahmen stereotaktischer Eingriffe mittels gezielter Tiefenhirnstimulation („Hirnschrittmacher“) zu behandeln. ${ }^{6}$ Kritiker dieser Initiative verweisen in der teilweise sehr emotional geführten Diskussion auf die Geschichte der psychiatrischen Chirurgie. Durch die bewusste Wahl des Reizworts „Psychochirurgie“ bzw. der ebenfalls negativ konnotierten (Teil-)Synonyme „Lobotomie“ bzw. „Leukotomie“ werden explizit Parallelen zu den oben skizzierten, eindeutig desavouierten Verfahren unterstellt. Aus medizinhistorischer und ethischer Sicht scheint eine solche Argumentation fragwürdig, da de facto weder in Bezug auf die Indikationsstellung und die Technik noch in Bezug auf die Invasivität, die Zielsetzung und die Nachbehandlung eine echte Vergleichbarkeit der rezenten Maßnahmen mit den „historischen“ psychochirurgischen Interventionen gegeben ist.

Wenngleich die skizzierte Diskussion mithin nicht sachlich geführt wird, erweist sie sich als höchst aufschlussreich: Sie lässt auf tiefe Ängste schließen, die sich in grundsätzlicher Weise mit der Rolle der Medizin als Deutungsmacht über Gesundheit und (therapiebedürftige) Krankheit und speziell mit Eingriffen in das Gehirn - also in die materielle Basis der Persönlichkeit, in das Zentrum von Kognition, Emotion, Gedächtnis und Handlungssteuerung ${ }^{7}$ - verbinden.

Im Rahmen dieses Beitrages soll gezeigt werden, dass die gegenwärtigen, mit diffusen Ängsten verknüpften Kritikpunkte auf einen Diskurs zurückgehen, der insbesondere in den 196oer und 1970er Jahren innerhalb der medizinischen Fachorgane und der fachnahen Presse geführt, zeitgleich bzw. zeitnah aber auch literarisch und filmisch verarbeitet wurde. Es soll insbesondere die Rolle der (verfilmten) belletristischen Literatur als Spiegel dieses Diskurses und des damit verbundenen Bildes der Psychochirurgie als Maßnahme der Restriktion und der sozialen Disziplinierung herausgearbeitet werden.

Folgende Vorannahmen bilden dabei den Ausgangspunkt des Beitrages: In der Literatur werden umstrittene medizinische Konzepte thematisiert und konfligierende Interessen und Positionen namhaft gemacht. Die Darstellung der Medizin in der Literatur reflektiert und prägt den gesellschaftlichen Blick auf Medizin ${ }^{8}$ - und hier insbesondere auf die psychiatrische Chirurgie -, d. h.

5 Vgl. Groß (2007).

6 Vgl. exemplarisch Albrecht (2004a); Albrecht (2004 b); Adler (2004); Voderholzer (2004); Röckerath (2004). Vgl. auch (insb. für den Diskurs in Frankreich) Groß (2007).

7 Müller (2007), S. 175.

8 Zum Verhältnis von Literatur und Medizin aus literaturwissenschaftlicher Sicht vgl. Müller-Seidel (1997); Anz (1989); Erhart (1997); Erhart (2004); Thomé (1993); Schnitzler (1988). Aus medizinhistorischer Sicht vgl. neben lagow/Steger (2004, 2005 und 2006) insb. Engelhardt (1991 und 2000). International sind v. a. die Studien von Sander L. Gilman anzuführen, hier insbesondere: Gilman (1988). Vgl. ferner Sontag (1977 und 1988). 
Literatur bildet Meinung ab und wirkt zugleich meinungsbildend. $\mathrm{Zu}$ untersuchen sind demnach Austauschprozesse zwischen der fachlichen und der gesellschaftlichen Diskussion über Psychochirurgie, deren Verarbeitung in der (verfilmten) belletristischen Literatur sowie die zeitgenössische gesellschaftliche Wahrnehmung von neurochirurgischen Eingriffen zur Behandlung psychiatrischer Störungen. Der vorliegende Beitrag geht damit von der Hypothese aus, dass die Ängste vor einer „Rückkehr der Psychochirurgie“9 nicht zuletzt durch die literarischen und filmischen Darstellungen psychiatrischer Maßnahmen geprägt sind: ${ }^{10}$ Da die meisten Menschen zeitlebens keine persönlichen Erfahrungen mit der Anstaltspsychiatrie machen, beruht das Bild der psychiatrischen Kliniken in besonderem Maße auf medialen Darstellungen.

Auf der Grundlage dieser Vorannahmen sollen das Bild der Psychiatrie und die Wahrnehmung des psychisch Kranken in ihrer literarischen und filmischen Konstruktion schrittweise herausgearbeitet werden. Zu diesem Zweck werden vier beispielhafte Fachtexte mit vier ebenfalls exemplarisch ausgewählten literarischen Werken bzw. deren filmischen Adaptationen kontrastiert, wobei der zeitliche Schwerpunkt bei beiden Textkorpora auf den 196oer und 1970er Jahren liegt. Dabei möchten wir zunächst den wissenschaftlichen Diskurs über die Psychochirurgie in den 196oer und 1970er Jahren nachzeichnen (Kapitel 2), um uns dann der Verarbeitung dieses Diskurses in der belletristischen Literatur zuzuwenden (Kapitel 3). Am Ende stehen einige kurz gefasste Schlussfolgerungen (Kapitel 4).

\section{Der fachliche bzw. fachnahe Diskurs über die hirnchirurgische Behandlung psychisch Kranker in den 1960er und 1970er Jahren}

Spätestens zu Beginn der 196oer Jahre gewann die fachliche Auseinandersetzung um die Zulässigkeit und den adäquaten Einsatz der Psychochirurgie an Schärfe. Sie wurde zunächst vorrangig fachintern geführt, erreichte jedoch bald auch wissenschaftsjournalistische Kreise. In der Mitte der 1970er Jahre wurde der Diskurs über das Für und Wider der Psychochirurgie dann öffentlich ausgetragen, wobei der Meinungsstreit nicht immer sachlich verlief. ${ }^{11}$ Dieser Diskurs soll im Folgenden am Beispiel von vier fachnahen Texten ${ }^{12}$ skizziert werden, wobei vor allem die Bedenken an dem Verfahren nachzuzeichnen sind.

Ausgangspunkt ist ein sehr differenzierter und ausgewogener Fachbeitrag von Siegfried Haddenbrock, der sich 1961 im „Handbuch für Neurosenlehre und Psychotherapie“ mit der Psychochirurgie auseinandersetzte. ${ }^{13}$ Haddenbrock beginnt mit der Feststellung, dass der „Wesensfrage: was tue ich wem und womit" ${ }^{\text {it }}$ im Hinblick auf die Psychochirurgie besondere Bedeutung zukomme, „wagt sich doch der Arzt daran, durch Ausschaltung gesunden Hirngewebes

\footnotetext{
9 Vgl. die Überschrift bei Albrecht (2004a).

10 Maio (2004), S. 153.

11 Vgl. exemplarisch die Ausführungen des Sexualwissenschaftlers Volkmar Sigusch in Kapitel 3 des vorliegenden Beitrages: Sigusch (1977), S. 25.

12 Folgende vier fachnahe Texte werden hierbei zugrunde gelegt: Haddenbrock (1961); Koch (1976); Sigusch (1977); Adler/Saupe (1979).

13 Vgl. Haddenbrock (1961).

14 Haddenbrock (1961), S. 35.
} 
Manifestationsmöglichkeiten einer menschlichen Person in ihrer leiblich-seelisch-geistigen Totalität einzuengen [...] “. ${ }^{15}$ Er gibt weiterhin zu bedenken, dass „ein therapeutischer Effekt nur um den Preis einer günstigenfalls minimal zu haltenden regressiven Persönlichkeitsveränderung [...] zu erzielen“ sei. ${ }^{16}$ Haddenbrock verurteilt psychochirurgische Eingriffe bei chronischen Zwangsneurosen, ${ }^{17}$ wobei er auf die schwerwiegenden Folgen der Frontotomien verweist: „[...] für die Frontalversehrten sind Angst und Sorge keine Grundbefindlichkeiten mehr: sie sind kein Dasein mehr, das sich in seinem Sinn verstehend zu diesem Sein verfällt [...] es fehlt den Kranken ,Eigentlichkeit', sie sind an das ,Man', an die Umwelt ,verfallen“." ${ }^{18}$ Auch die „Frontotomie psychopathischer Persönlichkeiten, insbesondere zur Verhinderung von Rückfallskriminalität“ ist nach Haddenbrock „eine nur in den seltensten Fällen gerechtfertigte Indikation. “19 Eine rein „soziale Indikation“ für Lobotomien lehnt er ebenfalls entschieden ab. ${ }^{20}$ Insgesamt redet Haddenbrock allerdings nicht einer „völligen Ablehnung der Methode“ das Wort, sondern fordert vielmehr „eine sehr sorgfältige Untersuchung, ob die Persönlichkeit des Kranken [...] mehr durch sein Leiden oder mehr durch den irreversiblen Eingriff beeinträchtigt wird. “21

Der Wissenschaftsjournalist Egmont Koch warnt einige Zeit später in einer Buchveröffentlichung mit dem werbewirksamen Titel „Chirurgie der Seele. Operative Umpolung des Verhaltens“ ebenfalls vor einer voreiligen Anwendung des Verfahrens. Er vertritt die „Auffassung, dass diese Therapie zwar prinzipiell berechtigt ist, aber bei der gegenwärtig praktizierten Handhabung eine Reihe schwerwiegender Probleme aufweist. Psychochirurgie darf kein Spielzeug für übereifrige Hirnoperateure sein. “22 Koch verweist vor allem auf Verfehlungen in den Anfangszeiten der Psychochirurgie und spekuliert, dass die moderne, verfeinerte stereotaktische Technik $^{23} \mathrm{zu}$ einer allzu großzügigen Indikationsstellung führen könnte:

„Die Leukotomie-Ära der vierziger und fünfziger lahre, während der auch Ehebruch und unregelmäßiger Stuhlgang als Indikation für messerchirurgische Hirnverstümmelungen galten, droht sich in gewisser Weise zu wiederholen. Denn dadurch, dass sich die Technik heute erheblich verfeinert hat, legen manche Operateure schon wieder zum Teil unkritisch, mitunter leichtsinnig, manchmal einer diffusen Ideologie der Behandlung allen psychischen Übels folgend, Hand an verhaltenskranke Patienten. ${ }^{\text {(24 }}$

15 Haddenbrock (1961), S. 35. Kursivierung durch den Verfasser Haddenbrock.

16 Haddenbrock (1961), S. 43.

17 Heute spricht man von Zwangsstörungen; vgl. Haddenbrock (1961), S. $45 \mathrm{ff}$.

18 Ebd.

19 Haddenbrock (1961), S. 54.

20 Haddenbrock (1961), S. 56.

21 Ebd.

22 Koch (1976), S. 8.

23 Die stereotaktische Methode, die 1948 in Lissabon vorgestellt worden war, zeichnet(e) sich durch das gezielte Einführen von Instrumenten in das Gehirn nach vorheriger stereometrischer Bestimmung und Errechnung eines Zielgebietes aus. Stereotaktische, also im Raum gerichtete Verfahren erlaubten es fortan, kleinere, umschriebenere Hirnareale anzuzielen und die Läsionen begrenzt zu halten. Allerdings durchlief die Technik ein längeres Erprobungsstadium, so dass zumindest bis zur Mitte der 1950er Jahre die Leukotomien und die Lobotomien dominierten: vgl. Henschen/Klingler/Riechert (1953), S. 552; für Details vgl. Adler/Saupe (1979), S. 78-81.

$24 \operatorname{Koch}(1976)$, S. 8. 
Kochs Kritik gipfelt in der Forderung, dass „nicht die wissenschaftliche Neugier, der experimentelle Spieltrieb oder gar der Ehrgeiz der Psychochirurgen“, sondern ausschließlich das Patientenwohl im Mittelpunkt der Überlegungen stehen müssten. Daher schlägt er vor, „die Fortführung psychochirurgischer Operationen in der Bundesrepublik an bestimmte Forderungen“ zu knüpfen. ${ }^{25}$ Koch beendet seine Ausführungen mit einem Seitenhieb gegen die psychochirurgisch tätigen Operateure: „Was beunruhigt, ist tatsächlich weniger die Psychochirurgie, sondern sind vielmehr die Männer, die sie machen. “ ${ }^{26}$

Während Egmont R. Koch die Anwendung der Psychochirurgie an bestimmte Bedingungen knüpfen möchte, wendet sich der Arzt und Sexualwissenschaftler Volkmar Sigusch in seinem Beitrag „Medizinische Experi-

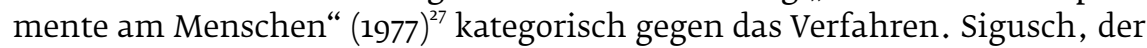
gewissermaßen als Wortführer der deutschen Lobotomie-Gegner gelten kann, spricht mit Blick auf die Psychochirurgie von einem „Massenexperiment“ ", ${ }^{28}$ sieht hierin „ein Beispiel enormer Brutalität“" ${ }^{\text {“29 }}$ und konstatiert ein „Absinken ins Subhumane“. ${ }^{30}$ Er verweist vor allem auf den sanktionierenden Charakter der Intervention bei sozial unerwünschtem Verhalten. Den Operateuren unterstellt er „therapeutische Raserei“, „Experimentierfreudigkeit“ und „Karrierismus“. ${ }^{31}$ Siguschs vernichtende Kritik an psychochirurgischen Eingriffen kulminiert in folgender Stellungnahme:
„Psychochirurgische Hirnoperationen sind Vernichtungs-Therapie‘. Sie werden am gesun- den Gehirn vorgenommen. Sie setzen bleibende anatomische und geistig-seelische Defekte, hinter die kein Weg zurückführt. Sie sind keine Notfalltherapie zur Erhaltung menschlichen Lebens. Sie sind weder mit der Amputation einer Extremität noch mit der chirurgischen Entfernung eines krebstragenden Organs noch mit Kastrationen gleichzusetzen. Sie besei- tigen und verändern höchste geistige, emotionale und soziale Fähigkeiten des Menschen. Sie sind Eingriffe in die Integrität des Menschen, die in der Heilkunde ihresgleichen suchen. Von den Menschenversuchen faschistischer KZ-Ärzte abgesehen, können es nur die gegen- wärtig praktizierten elektronisch gesteuerten Hirnreizungen zur Kontrolle menschlichen Verhaltens mit ihnen aufnehmen - an Skrupellosigkeit, an politischem Missbrauch, an ge- sellschaftlicher Bedeutung. ${ }^{\text {"32 }}$

Als letzter fachnaher Diskussionsbeitrag sei die Monographie „Psychochirurgie“, die der Psychiater und Medizinprofessor Meinhard Adler und der Geisteswissenschaftler Rolf Saupe in gemeinsamer Autorenschaft im Enke-Verlag veröffentlichten, angeführt. Sie erschien 1979 rund 18 Jahre nach dem Übersichtsartikel von Haddenbrock. Adler und Saupe, die auch die internationale Diskussion rezipieren und nachzeichnen, betonen ihr Bemühen um eine ausgewogene und unvoreingenommene Argumentation und äußern eingangs

\footnotetext{
25 Koch (1976), S. 208-211.

26 Ebd.

$27 \mathrm{Vgl}$. Sigusch (1977).

28 Ebd.

29 Ebd.

30 Sigusch (1977), S. 13.

31 Sigusch (1977), S. 18.

32 Sigusch (1977), S. 24 f.
} 
die Hoffnung, „dass unsere Darstellung einem mündigen Leser zu einer begründeten Stellungnahme verhilft. "33 Sie vertreten den Standpunkt, dass die Debatte um die Psychochirurgie in beiden Lagern über weite Strecken unsachlich geführt wird:

„Die Weigerung, die sachliche Basis der Psychochirurgie anzuerkennen, und eine abstrakte Fetischisierung der Allmacht der Technik auf der einen Seite, die Weigerung, das eigene Wirken in einen Kontext explizierter gesellschaftlicher Verantwortung zu stellen und die Weigerung, den Einsatz und die Effekte dieser Therapietechnik methodisch zu reflektieren, sind die Charakteristika der Pole in dieser Debatte. ${ }^{\text {"34 }}$

Adler und Saupe sprechen sich letztlich gegen ein „totales Verbot der Psychochirurgie" aus:

„Es muß allerdings sichergestellt werden, dass ein solches Verfahren weder einseitig noch überflüssig noch extensiv angewandt wird; aber wenn es hilft, soll derjenige, der es braucht, das Recht haben, es zu bekommen. ${ }^{\text {“35 }}$

Gleichzeitig räumen die Autoren ein, es gäbe „Hinweise auf einen möglichen Missbrauch und eine unklare Indikationsstellung, die eine schärfere Formulierung der Standards psychochirurgischer Praxis notwendig erscheinen lassen." ${ }^{36}$

\section{Die Verarbeitung des Diskurses über die hirnchirurgische Behandlung psychisch Kranker in fiktionalen Texten}

Wenden wir uns nun der Frage zu, ob, inwieweit bzw. auf welche Weise das in medizinischen und medizinjournalistischen Kreisen höchst strittige Thema Psychochirurgie in der belletristischen Literatur der 196oer und 1970er Jahre reflektiert und verarbeitet wird. Folgende vier literarische Werke ${ }^{37}$ sollen hierbei im Hinblick auf die skizzierte Fragestellung näher untersucht werden: Ken Keseys Bestsellerroman „One flew over the Cuckoo's Nest“ (Deutscher Titel: „Einer flog über das Kuckucksnest“), Michael Crichtons „The Terminal Man“ („Endstation“), William Arnolds fiktionale Biographie „Shadowland“ und Janet Frames autobiographisch geprägte Werke „Faces in the Water“ („Gesichter im Wasser“) und „An Angel at my Table“ („Ein Engel an meiner Tafel“).

Wenn die eingangs formulierte These, wonach Literatur den gesellschaftlichen Blick auf Medizin widerspiegelt und mitbestimmt, zutreffen sollte, müssen drei Forderungen erfüllt sein:

1. Der untersuchten belletristischen Literatur ist eine gewisse Reichweite und Popularität zuzuschreiben, d.h. sie wird nicht nur innerhalb der

33 Adler/Saupe (1979), S. X.

34 Adler/Saupe (1979), S. 245.

35 Adler/Saupe (1979), S. 251.

36 Adler/Saupe (1979), S. 259.

37 Kesey (1962) (verfilmt 1975 sowie 1978); Crichton (1972) (verfilmt 1974); Arnold (1978) (verfilmt 1982 unter dem Titel Frances); Frame (1961), sowie Frame (1984) (verfilmt 1990). 
Scientific Community rezipiert, sondern öffentlich wahrgenommen, denn nur dann kann sie eine meinungsbildende Wirkung entfalten.

2. Der eigentliche fachliche Diskurs über die operative Behandlung psychisch Kranker in der untersuchten belletristischen Literatur stehen in einem erkennbaren zeitlichen Zusammenhang und finden im selben sozialen Kontext bzw. in ähnlichen gesellschaftlichen Kontexten statt.

3. Die untersuchte belletristische Literatur nimmt inhaltlich klar erkennbar auf diesen fachlichen Diskurs Bezug und reflektiert diesen.

Der Nachweis, dass es sich bei den vier nachfolgend zitierten Werken um wirkmächtige Beispiele handelt, ist vergleichsweise leicht zu führen:

(1) Ken Kesey erzielte mit dem 1962 veröffentlichten Roman „One flew over the Cuckoo's Nest" einen Welterfolg. ${ }^{38}$ Bis 2002 wurde das Werk mehr als acht Millionen Mal verkauft. Er gilt als einer der wichtigsten Romane der US-amerikanischen Nachkriegszeit und war über Jahrzehnte hinweg in vielen amerikanischen Schulen Bestandteil des Lehrplans. ${ }^{39}$ Das Werk wurde 1963 von dem renommierten Dramatiker Dale Wasserman zu einem ebenfalls sehr erfolgreichen gleichnamigen Theaterstück umgeschrieben. Die bemerkenswerte Resonanz auf den Roman weckte zudem das Interesse Hollywoods an einer Verfilmung: Der Film erschien nach mehreren vergeblichen Drehbuchadaptationen 1975, also dreizehn Jahre nach der Veröffentlichung des Romans, unter der Regie von Miloš Forman und wurde ebenfalls ein herausragender künstlerischer und kommerzieller Erfolg. ${ }^{40}$ Er gewann fünf Oscars, u. a. für den besten Hauptdarsteller, Jack Nicholson, der die Person des lobotomierten Kleinkriminellen Randle Patrick (R. P.) McMurphy verkörperte und von seinem Publikum bis zum heutigen Tag in einen gedanklichen Zusammenhang mit dieser Kunstfigur gestellt wird. Kaum eine filmische Figur dürfte das Bild des lobotomierten Patienten in der Öffentlichkeit so sehr geprägt haben wie R. P. McMurphy alias Jack Nicholson.

(2) Wenngleich das literarische Oeuvre des US-amerikanischen Schriftstellers, Drehbuchautors und Regisseurs Michael Crichton eher der Unterhaltungs- als der Weltliteratur zuzurechnen sind, gehören seine Romane zu den erfolgreichsten und populärsten ihres Genres. Die Gesamtauflage der von Crichton verkauften Bücher beläuft sich auf 150 Millionen; seine Werke werden in 36 Sprachen übersetzt. Crichton schrieb u. a. den Erfolgsroman Jurassic Park und das Drehbuch zum gleichnamigen Steven-Spielberg-Film, der zu den Klassikern der kommerziellen Filmgeschichte zählt. Crichton wird eine besondere Affinität zu medizinischen Themen nachgesagt. ${ }^{41}$ Wie nahezu alle Werke von Crichton erzielte auch der hier analysierte Roman „The Terminal Man“(„Endstation“), der einen hirnchirurgischen Eingriff an dem Verbrecher Harry Benson in den Mittelpunkt der Handlung stellt, eine breite Öffentlichkeit. „The Terminal Man“ erschien bereits vor der Veröffentlichung im Buch-

38 Vgl. http://de.wikipedia.org/wiki/Ken_Kesey; http://en.wikipedia.org/wiki/Ken_Kesey.

$39 \mathrm{Vgl}$. www.litencyc.com/php/speople.php?rec=true\&UID=4941.

$40 \mathrm{Vgl}$. http://de.wikipedia.org/wiki/Einer_flog_\%C3\%BCber_das_Kuckucksnest.

41 So gilt er u. a. als Schöpfer der erfolgreichen TV-Arztserie „Emergency Room“. 
handel in gekürzter Version im amerikanischen Playboy. ${ }^{42}$ Auch „The Terminal Man" wurde 1974 in den USA verfilmt; allerdings konnte der Regisseur Mike Hodges trotz prominenter Besetzung ${ }^{43}$ und der Mitwirkung von Crichton am Drehbuch hiermit keinen durchschlagenden Erfolg verbuchen. ${ }^{44}$

(3) Große Resonanz erzielte auch die fiktionale Biographie „Shadowland“ des US-amerikanischen Filmkritikers William Arnold aus dem Jahr 1978. Arnold zeichnete in Shadowland das Leben der Hollywood-Schauspielerin Frances Farmer nach, die 1943 nach diversen Skandalen für geisteskrank erklärt und in eine Nervenheilanstalt eingeliefert worden war. Besondere Aufmerksamkeit erzielte Arnold mit der fälschlichen Behauptung, die Schauspielerin sei während ihres elfjährigen Aufenthaltes in der Heilanstalt lobotomiert worden. ${ }^{45}$ Auch Arnolds auf Publikumswirksamkeit angelegtes Werk zog das Interesse der Filmindustrie auf sich und wurde schließlich 1982 unter dem Titel „Frances“verfilmt, wobei man an der Lobotomie-Sequenz trotz ihres bekanntermaßen fiktionalen Charakters festhielt. Die von dem Regisseur Graeme Clifford verantwortete Verfilmung wurde ebenfalls ein weltweiter Erfolg: Der Film wurde für zwei Oscars nominiert, u. a. für die Rolle des Frances Farmer, die von der Schauspielerin Jessica Lange verkörpert wurde. Buch und Film sorgten für ein bemerkenswertes Interesse am Schicksal von Farmer, das selbst die Jugendbewegung und -kultur erfasste: Als markantes Beispiel sei die international erfolgreiche Rockgruppe Nirvana erwähnt, die auf ihrem 1993 publizierten Album „In Utero“ in Anspielung auf die Erlebnisse der Schauspielerin in der damaligen Anstaltspsychiatrie einen Song mit dem Titel „Frances Farmer will have her revenge on Seattle" präsentierten. ${ }^{46}$

(4) Die 2004 verstorbene neuseeländische Schriftstellerin Janet Frame zählte ihrerseits zu den bedeutendsten Literatinnen des 2o. Jahrhunderts. Frame wurde für ihr belletristisches Werk vielfach ausgezeichnet und war 2003 eine Anwärterin auf den Literaturnobelpreis. In ihren beiden autobiographischen Romanen „An Angel at my Table“ (1984) und insbesondere „Faces in the Water“ (1961) thematisiert sie die Schrecken ihres insgesamt achtjährigen Aufenthalts in psychiatrischen Kliniken. Besonders eindrücklich beschreibt Frame, bei der in jungen Jahren die Krankheit Schizophrenie diagnostiziert worden war, die Angst vor den regelmäßigen Elektroschock-Therapien und der abschließend geplanten Lobotomie, welcher sie jedoch in letzter Sekunde unter kuriosen Umständen entkommen konnte. ${ }^{47}$ Auch die autobiographischen Erinnerungen von Frame zogen das Interesse der Filmindustrie nach sich: 1990 verfilmte die Regisseurin Jane Campion ${ }^{48}$ Frames Leben („An Angel at my Table“) - mit durchschlagendem Erfolg, denn der Film gewann u. a. auf dem Internationalen Film Festival von Venedig sieben Preise einschließlich des Spezialpreises

42 Vgl. hierzu Crichton (1973), S. 4.

43 Die Hauptrolle hatte der bekannte Filmschauspieler George Segal übernommen:

vgl. www.imdb.com/name/nm0001719/; www.imdb.com/title/tt0072267/.

44 Vgl. www.imdb.com/title/tt0072267/.

$45 \mathrm{Vgl.} \mathrm{hierzu} \mathrm{http://jeffreykauffman.net/francesfarmer/sheddinglight.html.}$

$46 \mathrm{Vgl}$. http://de.wikipedia.org/wiki/Frances_Farmer. Siehe auch Borzillo-Vrenna (2004).

47 Vgl. hierzu Kapitel 3 dieses Beitrages sowie Frame (1993), S. 295.

48 Jane Campion hat 1993 mit dem Kinofilm „Das Piano“ einen weiteren weltweiten Erfolg verbucht. 
der Jury sowie den „The Four Season’s International Critics Award“ auf dem Filmfestival von Toronto. ${ }^{49}$

Aus den bisherigen Ausführungen ergibt sich, dass die zitierten literarischen Beispiele nicht nur in einem kleinen Gelehrtenkreis rezipiert wurden, sondern ein vergleichsweise breites Publikum erreichten; besonders anschaulich wird dieses Faktum in Anbetracht der Tatsache, dass alle angeführten Werke nachfolgend verfilmt wurden. Damit kann nunmehr der Frage nachgegangen werden, ob der in der Scientific Community bzw. in fachnahen Kreisen (Wissenschaftsjournalismus) geführte Diskurs über Psychochirurgie und die Thematisierung desselben in der untersuchten (fiktionalen) Literatur in zeitlichem Zusammenhang standen und in gleichen bzw. vergleichbaren sozialen Kontexten stattfanden:

Die vier zugrunde gelegten fachnahen Texte zum Thema Psychochirurgie sind zwischen 1961 und 1979 entstanden, und die literarischen Texte sind ebenfalls dem Zeitraum zwischen 1962 und 1978 zuzuordnen. ${ }^{50}$ Beide Textkorpora (fachnahe Texte und fiktionale Literatur) stehen somit in zeitlicher Nähe zueinander. Auch konnte bereits gezeigt werden, dass die zugrunde gelegte belletristische Literatur und deren filmische Adaptationen international rezipiert wurden, d. h. die Werke wurden in ihren jeweiligen Übersetzungen in der Bundesrepublik Deutschland ebenso wahrgenommen wie in den europäischen Nachbarstaaten oder in Übersee. Gleiches gilt für den fachlichen Diskurs über Psychochirurgie, wie Adler und Saupe schlüssig nachgewiesen haben. ${ }^{51}$ Damit ist sichergestellt, dass beide Textkorpora nicht nur in zeitlicher Nähe stehen, sondern auch (ungeachtet der unterschiedlichen Herkunftsländer der Autoren) unter vergleichbaren gesellschaftlichen Rahmenbedingungen entstanden sind.

Bleibt die zentrale Forderung, dass die untersuchte belletristische Literatur inhaltlich klar erkennbar auf diesen fachlichen Diskurs Bezug nimmt, ihn reflektiert und so meinungsbildend wirkt. Dieser Aspekt soll im Folgenden näher betrachtet werden: Im Roman „Einer flog über das Kuckucksnest“ lässt sich der Kleinkriminelle Randle Patrick McMurphy in eine psychiatrische Anstalt einliefern, um einer Gefängnisstrafe zu entgehen. McMurphy will sich der Anstaltsroutine nicht unterwerfen, sondern rebelliert gegen das System, von dem die Insassen u.a. mit Medikamenten, Elektroschocks und psychochirurgischen Maßnahmen ruhig gestellt werden. Wegen seines unnachgiebigen Widerstandes gegen die Unterdrückung in Misskredit geraten, wird McMurphy schließlich nach einem tätlichen Angriff gegen eine hartherzige Schwester namens Ratched in einem Akt der Willkür einer Lobotomie unterzogen, die einen schweren Hirnschaden verursacht und ihn seiner Per-

49 Vgl. www.imdb.com/title/tt0099040/www.sensesofcinema.com/contents/directors/02/campion.html. www.djflde/entertainment/stars/j/jane_campion.html.

50 Eine Ausnahme bildet die Autobiographie „An Angel at my Table“ (1984), die jedoch inhaltlich auf „Faces in the Water" (1961) rekurriert. Demgegenüber sind die diversen filmischen Adaptationen der genannten Werke naturgemäß jüngeren Datums als die eigentliche belletristische Literatur.

51 Die Autoren verweisen darauf, dass die Debatte in den USA begann und dann nach Europa gelangte: Adler/Saupe (1979), S. 245. Zudem widmen sie ein Kapitel der Diskussion um die Psychochirurgie im internationalen Vergleich (S. 246-249). 
sönlichkeit beraubt. Keseys Roman basiert auf Erfahrungen, die der Schriftsteller 1959 machte, als er am Veterans Hospital im kalifornischen Menlo Park als Aushilfe in der dortigen Psychiatrieabteilung tätig war. Hier wuchs seine Überzeugung, dass viele Patienten nicht geisteskrank waren, sondern wegen sozialer Missliebigkeit eingewiesen wurden. Kesey beschreibt die Anstalt als Ort der Repression. Die Pflegekräfte werden als verschlagen, empathielos und inhuman charakterisiert, etwa, wenn es über die zuständige Schwester Ratched heißt:

„,... sie muß sich schnell wieder zurückverwandeln, bevor ihre derzeitige Verfassung ihr tatsächliches Ich in seiner ganzen Häßlichkeit für alle sichtbar macht [...] Ihr Gesicht ist glatt, berechnend, eine präzise Spezialanfertigung. ${ }^{452}$

Psychochirurgische Eingriffe kommentieren die Insassen wie folgt:

„, Lobotomie, ist das nicht die Sache, wo sie einem das halbe Hirn wegschneiden?' ,Ganz richtig. Sie sind allmählich recht gut bewandert in unserem Kauderwelsch. Jawohl, wo sie einem das halbe Hirn wegschneiden. Stirnlappen-Kastration. Wenn sie das Messer nicht unter der Gürtellinie ansetzen kann, dann macht sie es eben über den Augen. “" ${ }^{\text {(33 }}$

Nachdem McMurphy selbst einer Lobotomie unterzogen wurde, wird er von seinen Mitpatienten in Augenschein genommen. Dabei entwickelt sich folgender Dialog:

„,Aber den Gesichtsausdruck, das geht nicht. Da ist überhaupt nichts in dem Gesicht. Grad wie eine Schaufensterpuppe, hab ich recht, Scanlon?'Scanlon spuckte wieder auf den Boden. ,Ganz klar, verdammt. Das Ganze ist, na ja, zu leer. Das sieht doch jeder. “" ${ }^{\text {c54 }}$

Wenig später wird das ganze Ausmaß der Vernichtung sichtbar:

„Die Schwellungen um die Augen waren so weit zurückgegangen, dass sie offen waren; sie starrten in das volle Licht des Mondes, offen, nicht träumend, ganz glasig, weil sie schon so lange ohne Zwinkern offen waren, bis sie wie durchgebrannte Sicherungen aussahen."“55

Schließlich wird McMurphy von einem Mitpatienten, der die Konfrontation mit dem postoperativen Zustand des Lobotomierten nicht erträgt, erstickt. ${ }^{56}$ Psychochirurgie ist bei Kesey ein Symbol für den repressiven Charakter der Anstaltspsychiatrie, die für Schrecken und Unmenschlichkeit steht. Sie wird angewandt, um unerwünschtes Sozialverhalten zu sanktionieren. Ebendiese „soziale Indikation“ der Psychochirurgie, die in Siguschs Kritik eine zentrale Rolle spielt, steht auch bei Kesey im Vordergrund.

Ähnlich vernichtend fällt die Schilderung eines hirnchirurgischen Eingriffes in Michael Crichtons Roman „Endstation“ aus. Der Protagonist Harry

\footnotetext{
52 Kesey (1996), S. $11 \mathrm{f}$.

53 Sigusch (1977), S. 200.

54 Sigusch (1977), S. $339 \mathrm{f}$.

55 Crichton (1973), S. $340 \mathrm{f}$

56 Ebd.
} 
Benson leidet seit einem Autounfall unter psychomotorischer Epilepsie, die ihn aggressiv und gewalttätig werden lässt, so dass er zwei Mordversuche unternimmt. ${ }^{57}$ Dr. Roger McPherson, leitender Arzt der neuropsychiatrischen Abteilung, will Benson, der sich zunächst kooperativ zeigt, einer Hirnoperation unterziehen, bei der ferngesteuerte Elektroden implantiert werden sollen mit dem Ziel, die psychischen Auffälligkeiten zu kontrollieren. Anders als bei den übrigen literarischen Quellen ist der Eingriff im vorliegenden Fall mit einer Implantation verknüpft. Janet Ross, Psychologin im Krankenhaus, äußert im Vorfeld Bedenken gegenüber der Operation:

„Sie hatte Ellis ihre Meinung schon zu oft mitgeteilt. Es konnte sein, dass die Operation nicht nur nicht half, sondern Bensons Zustand sogar noch verschlimmerte. Sie war sich sicher, dass Ellis sich auch über diese Möglichkeit im Klaren war, sie jedoch starrköpfig ignorierte. “58

Tatsächlich setzt sich der zuständige Arzt über die Bedenken hinweg. Zunächst scheint der Eingriff erfolgreich zu verlaufen, doch dann stellen sich erste Komplikationen ein, durch die sich Ross bestätigt fühlt:

„, Ich habe ihnen von Anfang an gesagt - wirklich, von Anfang an -, dass es keine gute Idee sei, aber Ellis, Morris und McPherson waren erpicht darauf. Sie sind so hochnäsig, insbesondere Morris. Als ich inn in der Wachstation sah, wie er sich an Benson ergötzte - der war eingewickelt und bleich im Gesicht -, da wurde ich wütend. ““59

Benson selbst reagiert zunehmend verängstigt und aggressiv. Dem Arzt gegenüber gibt er an: „Ich komme mir vor wie eine verdammte Maschine. Wie ein Auto in einer komplizierten Werkstatt. "60 Benson findet heraus, wie er die Funktion der Elektroden selbst steuern kann, und flüchtet aus dem Krankenhaus. Er fühlt sich von den Ärzten betrogen ${ }^{61}$ und droht mit Rache:

„,Ihr kümmert euch um mich.' Sein Lachen war laut und hässlich. ,Ihr kümmert euch doch nicht um mich, sondern nur um euer Experiment. Das einzige, was euch kümmert, ist euer wissenschaftliches Protokoll. Ich selbst bin euch doch gleichgültig.' Er war jetzt aufgeregt und böse. ,Es sieht in den medizinischen Fachzeitschriften bestimmt nicht gut aus, wenn ihr berichten müsst, dass ihr jahrelang so viele Patienten beobachtet habt, und schließlich starb einer, weil er durchdrehte und die Bullen ihn niederknallten. Das macht einen schlechten Eindruck. “62

Am Ende wird Benson, dessen Aggressionen außer Kontrolle geraten, tatsächlich in Notwehr von der Psychologin Ross erschossen. ${ }^{63}$ Wie bei Kesey, so wird der operative Eingriff auch bei Crichton als Maßnahme geschildert, die dem Patienteninteresse und sozial verantwortlichem Handeln zuwider läuft. Steht

\footnotetext{
57 Crichton (1973), S. $57 \mathrm{f}$.

58 Crichton (1973), S. 45.

59 Crichton (1973), S. 112.

60 Crichton (1973), S. 159.

61 Crichton (1973), S. $228 \mathrm{f}$.

62 Crichton (1973), S. 232.

63 Crichton (1973), S. $312 \mathrm{f}$.
} 
bei Kesey der repressive Charakter der Behandlungsmaßnahme im Vordergrund, ist es bei Crichton die Eitelkeit und das Geltungsstreben des zuständigen Arztes. In beiden Romanen führen die Eingriffe zu schweren Hirnschäden und letztlich zum Tod der Operierten.

Der US-Filmkritiker und Autor William Arnold schildert in seiner fiktionalen Biographie Shadowland das Leben der Filmschauspielerin Frances Farmer. Nach diversen Skandalen wurde sie 1943 unter anderem wegen ihrer radikalen politischen Ansichten für geisteskrank erklärt und in eine Nervenheilanstalt eingeliefert, aus der sie erst nach elf Jahren wieder entlassen wurde. Die Biographie und die hierauf beruhende Verfilmung des Werkes erregten vor allem deshalb großes Aufsehen, weil Arnold fälschlicherweise ${ }^{64}$ behauptet, die Schauspielerin sei während ihres Aufenthaltes in der Anstalt lobotomiert worden. In Arnolds Biographie dient die Lobotomie als ein wesentliches dramaturgisches Element; diese wird dem Biographen zufolge nicht von einem beliebigen Operateur, sondern von dem weltweit ebenso bekannten wie berüchtigten Psychochirurgen Walter Freeman vorgenommen:

„Like so many other psychiatrists, Freeman had long been fascinated by the case of Frances Farmer [...] Toward the end of this second visit - at the very end of 1948 - Freeman had Frances brought before him in a remote treatment room. She was placed on a table and administered electric shock until passed out. All the nurses and orderlies then filed out the room. No one will ever know exactly what happened next, but the overwhelming conclusion drawn by the people of Steilacoom at the time was that the doctor lifted her right eyelid and stuck a needle into her brain. ${ }^{65}$ Because when she came to, Frances Farmer was not the same person she had been, and she would never be the same person again. ${ }^{6{ }^{66}}$

Besondere Aufmerksamkeit widmet Arnold der Schilderung postoperativer Veränderungen in der Persönlichkeit der Schauspielerin:

„As she recuperated over the following month, her attitude miraculously began to change. All the old rebellion drained out of her. Her resistance crumbled. Her perpetual sarcasm disappeared. She was like a totally new and different person - a cowering and obedient version of her former self. She came to believe that she was guilty. ${ }^{\text {“67 }}$

Dass die Operation in den Augen des Autors eine zerstörerische Wirkung zeigte, ergibt sich aus folgenden Zeilen:

"Sometimes old friends stopped by to see her, and they say they found a humorless and burned-out shell, a frightening counterfeit of what Frances had once been [...]. ${ }^{68}$

Arnold schildert Farmer als Forschungsobjekt in der Hand des Psychochirurgen, als interessanten klinischen Fall, an dem sich die Möglichkeiten der psy-

$64 \mathrm{Vgl}$. hierzu http://jeffreykauffman.net/francesfarmer/sheddinglight.html.

65 Beschrieben wird hier die so genannte transorbitale Lobotomie, ein Verfahren, bei dem ein stilettoartiges Messer, ein sog. Leukotom, über die Orbita in das Frontalhirn eingeführt wurde.

66 Arnold (1978), S. 223.

67 Arnold (1978), S. 224

68 Arnold (1978), S. 226 
chiatrischen Chirurgie erproben und demonstrieren lassen. So zitiert er u. a. einen mysteriösen Gewährsmann, der ihm gegenüber glaubhaft versichert habe, das „Establishment“ der amerikanischen Psychiatrie habe Frances Farmer „neu erschaffen" ${ }^{609}$ wollen:

„He said they had wanted to demonstrate how effective psychiatry could modify the behavior of even the most notorious troublemaker [...] He sounded, frankly, rather paranoid on the subject. ${ }^{\prime 70}$

Auch Arnold skizziert folglich den Psychochirurgen - ähnlich wie Crichton als geltungssüchtigen Arzt, die Anstaltspsychiatrie - ähnlich wie Kesey - als Ort der Unterdrückung und der sozialen Disziplinierung und die Lobotomie ebenso wie Kesey und Crichton - als verfehlte Maßnahme mit destruktivem Charakter.

Während die Biographie von Arnold deutliche fiktionale Elemente enthält (und überdies über weite Strecken auf Sensation und Effekt setzt), beruhen die dichten und bildreichen Schilderungen von Janet Frame erklärtermaßen ${ }^{71}$ auf autobiographischen Erfahrungen der Autorin. Aufgrund der (retrospektiv umstrittenen) Diagnose Schizophrenie verbrachte Frame acht Jahre, von 1947 bis 1954, in Nervenheilanstalten, wo sie mit 200 qualvollen Elektroschocks „therapiert" wurde. Die Erinnerungen an diese Zeit verarbeitet sie 1961 in ihrem Roman „Faces in the Water“ („Gesichter im Wasser“). Dort beschreibt die Protagonistin Estina die Grausamkeiten, die ihr in der psychiatrischen Anstalt widerfahren. Besonders der Sadismus der Bediensteten, die „zu gehetzten, lustlosen Heuchlerinnen, die andere misshandeln", ${ }^{72}$ werden, nimmt in ihrer Schilderung breiten Raum ein. ${ }^{73}$ Zur Lobotomie an einer Bekannten vermerkt sie:

„Ich kannte Brenda noch von Station vier. Ich erinnere mich, dass sie eine der ersten war, die zum Zweck der Veränderung der Persönlichkeit operiert wurde [...] Und jetzt, fünf Jahre später, war sie auf Station zwei - nach einer zweiten Operation, die man, so schien es, in dem verzweifelten Versuch unternommen hatte, die allzu früh sichtbare und erschreckende Wirkung der ersten zu bessern. Sie erinnerte sich an mich. Ich versuchte, nicht zu weinen, als ich wahrnahm, in welchem Zustand sie sich befand. ${ }^{\text {“74 }}$

Von den vielen eigenen Elektroschock-Therapien berichtet sie,

„dass die Behandlung einem alles entreißt und einen allein und blind im Nichts zurücklässt, und man sucht tastend wie ein neugeborenes Tier die Stelle, die einem den ersten Trost spendet; dann erwacht man, klein und verängstigt, und die Tränen fließen unaufhörlich in namenlosem Leid. ${ }^{\text {"75 }}$

69 Arnold (1978), S. 217: „[...] the entire psychiatric establishment of the country had cooperated to ,remake' Frances Farmer."

70 Ebd.

71 Frame (1993) (wie Anm. 48), S. 253.

72 Frame (1994), S. 119.

73 Frame (1994), S. 23f., 55, 102; Frame (1993), S. 293.

74 Frame (1994), S. 168.

75 Frame (1994), S. 26. 
Besonders intensiv ist die Beschreibung der Gedanken, die sie beschleichen, als man ihr miteilt, dass sie für eine Lobotomie vorgesehen ist. Der zuständige Arzt, Dr. Stewart, führt das betreffende Gespräch:

„,Wir sehen sie gar nicht gerne hier', sagte er. ,Es gibt eine Operation, die die Persönlichkeit verändert und die Spannung mindert, und wir haben entschieden, dass es das Beste für Sie ist, sich dieser Operation zu unterziehen. Einer Ihrer Angehörigen wird für Sie unterschreiben. Wie haben Ihre Mutter zu einem Gespräch hergebeten.' [...] Sein Adamsapfel trat hervor wie ein Teil eines Leitungssystems seines Halses. Sein Gesicht war grau. , Das Internierungslager in Deutschland', dachte ich [...]. ${ }^{77^{6}}$

Der Hinweis, dass nicht die Patientin selbst, sondern die Mutter zu einem Aufklärungsgespräch gebeten wird, zeigt beispielhaft die paternalistische Haltung damaliger Psychochirurgen. Psychochirurgische Interventionen waren eng verbunden mit einem asymmetrischen geprägten Arzt-Patient-Verhältnis, das die Patientenautonomie missachtete.

Wenig später schildert Estina ihre tiefen Ängste vor dem Eingriff:

„Ich fühlte mich von den Arrangements, die für mich getroffen wurden, weit entfernt; als würde ich auf meinem Totenbett liegen und zusehen, wie andere in mein Haus eindringen und über meine Schätze verfügen, und als würde ich durch die halbgeschlossene Tür im Nebenzimmer den bereit gestellten Sarg, meinen letzten Bau, mein milchweißes Spinnwebennest zwischen zwei Felsen sehen. ${ }^{\text { }} 77$

Estina lässt keinen Zweifel an der vernichtenden Wirkung der Lobotomie:

„Ich werde erwachen und keinerlei Kontrolle über mich haben. Ich habe andere gesehen, die ins Bett machen, die vage, ausdruckslose Gesichter haben und über ein Sortiment von unwirklich lächelnden Mienen verfügen, für die es keinen Bedarf gibt. Ich werde ,rekonditioniert' - dieses Wort benutzt man für Lobotomiefälle. Rehabilitiert. Angepasst, mein Gehirn wird zurechtgestutzt und für die Gepflogenheiten der Welt zurechtgeschnitten [...] Was genau werden sie stehlen - die behutsamen Einbrecher, die sich an meinem Gehirn zu schaffen machen? Ich wusste, es gab kein Entrinnen, und dennoch schrie ich Hilfe, Hilfe, aber ich war in Mauern eingeschlossen, bis Dr. Portman mich hörte..$^{478}$

Tatsächlich entging Janet Frame alias Estina in letzter Sekunde der bereits beschlossenen Lobotomie: Ihre Geschichtensammlung „The Lagoon“ hatte den Hubert Church Memorial Award gewonnen, ein Vertreter des Krankenhauses las von der Auszeichnung und die Operation wurde abgesagt. ${ }^{79}$ Auch Janet Frame zeichnet in ihrer Autobiographie ein ausgesprochen negatives Bild der Lobotomie:

„Meine Freundin Nola, die unglücklicherweise keinen Preis gewonnen hatte, deren Name nicht in der Zeitung stand, hatte ihre Lobotomie und kam zurück ins Krankenhaus [...] Die ,Lo-

76 Frame (1994), S. 246.

77 Frame (1994), S. 248.

78 Frame (1994), S. 249-251.

79 Vgl. Frame (1993), S. $295 \mathrm{f}$. 
botomien ' wurden angesprochen, spazierengeführt, mit Make-up und geblümten Schals, die ihre geschorenen Schädel bedeckten, hübsch gemacht. Sie waren still, fügsam; ihre Augen waren groß und dunkel, und ihre Gesichter bleich, mit feuchter Haut. Sie wurden ,geschult', sich in die Alltagswelt ,einzufügen', die immer als ,Draußen' bezeichnet wurde; ,die Welt draußen.' Im Wirbel der Arbeit und des Personalmangels und des allzu langen Schulungsprozesses wurden die Lobotomien der Reihe nach zu den Opfern entzogener Aufmerksamkeit und entzogenen Interesses; der falsche Frühling wurde wieder zum Winter. ${ }^{\text {80 }}$

Ähnlich wie in den vorgenannten literarischen Beispielen rückt Frame die Perspektive der Psychiatriegeschädigten in den Vordergrund. Sie betont in ihren autobiographischen Werken ebenfalls die vernichtende Wirkung des Eingriffs auf die Persönlichkeit und beschreibt das Ausgeliefertsein, die Willkür der Behandler, die grobe Missachtung der Patientenautonomie und die Ohnmacht des psychisch Kranken im Anstaltsbetrieb. Besonders eindrücklich kommt das Gefühl der eigenen Handlungsunfähigkeit in der Reaktion Estinas auf die Absage der Operation zum Ausdruck:

„In jener Nacht weinte ich in meinem kleinen Zimmer und wartete auf Mr. Griffiths, um ihm von meinem Glück über die Begnadigung zu erzählen [...]. ${ }^{\text {‘1 }}$

\section{Schlussfolgerungen}

Die Auswertung der Textkorpora lässt vier wesentliche Schlussfolgerungen zu, die sich folgendermaßen zusammenfassen lassen:

Zum ersten existieren deutliche Verflechtungen und Austauschprozesse zwischen der eigentlichen medizinischen und gesellschaftlichen Diskussion über Psychochirurgie und deren Verarbeitung und Darstellung in der (verfilmten) belletristischen Literatur. Der Diskurs über die Zulässigkeit operativer Eingriffe bei psychisch kranken Menschen wird in der ausgewählten Literatur und in deren Adaptationen thematisch aufgegriffen und reflektiert.

Zum zweiten fällt auf, dass im literarischen Textkorpus eine sehr kritische Darstellung psychochirurgischer Maßnahmen überwiegt, die weitgehend aus der Perspektive der psychiatrischen Patienten verfasst ist und - anders als im fachnahen Diskurs - etwaige sachliche Argumente der Verfechter dieser Maßnahmen nicht berücksichtigt.

Zum dritten lässt sich zeigen, dass die fiktionale literarische und filmische „Repräsentation“ von Anstaltspsychiatrie und Psychochirurgie das in den 1970er Jahren vorherrschende gesellschaftliche Bild von Psychiatrie und Psychochirurgie als Ort bzw. Maßnahme der Restriktion und der sozialen Disziplinierung prägt - ein Bild, das bis in die Gegenwart nachwirkt. ${ }^{82}$ Dabei tritt die fiktional geprägte literarische „Repräsentation“ vielfach an die Stelle fehlender realer persönlicher Erfahrungen mit psychiatrischer Somatotherapie.

80 Ebd.

81 Frame (1993), S. 252. - Nachträgliche Hervorhebung durch die Autoren dieses Aufsatzes.

82 Ein Beispiel für diese Abwehrhaltung ist die voreilige und objektiv gesehen unzulässige Gleichsetzung der Tiefenhirnstimulation mit den historischen psychochirurgischen Maßnahmen. 
Schließlich lässt die vorwiegend aus der Patientensicht verfasste literarische Darstellung psychochirurgischer Interventionen auf das Vorliegen weit reichender und tiefer Ängste schließen, die sich mit Eingriffen in das Gehirn - also in die materielle Basis der Persönlichkeit - verbinden. Damit wird zugleich deutlich, dass der literarischen Analyse eine substantielle Bedeutung zukommt, wenn es darum geht, Fragen und Ergebnisse der modernen Neurowissenschaften und der Hirnforschung in ihren gesellschaftlichen und anthropologischen Auswirkungen aufzugreifen, auszuwerten und in einen soziokulturellen Kontext zu stellen.

\section{Literatur}

Adler (2004): Meinhard Adler, Stereotaxie. Rückschau, Deutsches Ärzteblatt 101 (2004), S. A-3020/B-2551/ $\mathrm{C}-2434$

Adler/Saupe (1979): Meinhard Adler, Rolf Saupe, Psychochirurgie. Zur Frage einer biologischen Therapie psychischer Störungen, Stuttgart 1979

Albrecht (2004 a): Bernhard Albrecht, Stereotaxie/Hirnschrittmacher. Rückkehr der Psychochirurgie, Deutsches Ärzteblatt 101 (2004), S. A-2594/B-2184/C-2098

Albrecht (2004 b): Meinhard Adler, Operationen gegen den inneren Zwang, Süddeutsche Zeitung Nr. 69, 23.3.2004

Antunes (2000): João Lobo Antunes, Psicocirurgia - uma história, in: ders. (Hrsg.), Numa cidade feliz, Lisboa, 3. Aufl., 2000, S. 225-248

Anz (1989): Thomas Anz, Gesund oder krank? Medizin, Moral und Ästhetik in der deutschen Gegenwartsliteratur, Stuttgart 1989

Arnold (1978): William Arnold, Shadowland, New York 1978

Board of Control (1947): Board of Control, His Majesty's Stationery Office, Prefrontal Leucotomy in 1000 cases, London 1947

Borzillo-Vrenna (2004): Carrie Borzillo-Vrenna, Kurt Cobain und Nirvana. Chronik, Höfen 2004

Crichton (1972): Michael Crichton, The Terminal Man, London 1972

Crichton (1973): Michael Crichton, Endstation, Zürich 1973

Engelhardt (1991): Dietrich von Engelhardt, Medizin in der Literatur der Neuzeit, Bd. 1, Hürtgenwald 1991 Engelhardt (2000): Dietrich von Engelhardt, Medizin in der Literatur der Neuzeit, Bd. 2, Hürtgenwald 2000 Erhart (1997): Walter Erhart, Medizingeschichte und Literatur am Ende des 19. Jahrhunderts, Scientia Poetica 1 (1997), S. 224-267

Erhart (2004): Walter Erhart, Medizin - Sozialgeschichte - Literatur, Internationales Archiv für Sozialgeschichte der deutschen Literatur 29 (2004), S. 118-128

Fortner (2004): Rainer Fortner, Egas Moniz - Leben und Werk unter besonderer Berücksichtigung der Leukotomie und ihrer ethischen Implikationen, Diss. med. Würzburg 2004

Fortner/Groß (2002): Rainer Fortner, Dominik Groß, Egas Moniz und die Leukotomie-Debatte unter besonderer Berücksichtigung des portugiesischsprachigen Schrifttums, Sudhoffs Archiv 86 (2002), S. 138-170

Frame (1961): Janet Frame, Faces in the Water, New York 1961

Frame (1984): Janet Frame, An Angel at my Table, Auckland 1984

Frame (1993): Janet Frame, Ein Engel an meiner Tafel, München 1993

Frame (1994): Janet Frame, Gesichter im Wasser, München 1994

Gilman (1988): Sander L. Gilman, Disease and Representation. Images of Illness from Madness to AIDS, Ithaca (NY) 1988

Groß (1999): Dominik Groß, Psychochirurgie und Ethik: Die operative Behandlung psychischer Störungen vom 19. Jahrhundert bis heute, Fortschritte der Medizin 117 (1999), Ergänzungsband I/10. April 1999, S. 1-4 
Groß (2007): Dominik Groß, Von der Topektomie bis zur Stereotaxie: Die Geschichte psychochirurgischer Interventionen, in: Dominik Groß, Sabine Müller (Hrsg.), Sind die Gedanken frei? Die Neurowissenschaften in Geschichte und Gegenwart, Berlin 2007, S. 144-174

Haddenbrock (1961): Siegfried Haddenbrock, Psychochirurgie, in: Viktor Frankl (Hrsg.), Handbuch für Neurosenlehre und Psychotherapie, München 1961, S. 34-58

Henschen/Klingler/Riechert (1953): Carl Henschen, I. Klingler, Traugott Riechert, Kraniocerebrale Korrelationstopographie thalamofrontaler Bahnen und gezielte Hirnoperationen. Kritische Reflexion zur Leukotomie, Langenbecks Archiv für klinische Chirurgie und Deutsche Zeitschrift für Chirurgie 273 (1953), S. 548-565

Jagow/Steger (2004): Bettina von Jagow, Florian Steger (Hrsg.), Repräsentationen. Medizin und Ethik in Literatur und Kunst der Moderne, Heidelberg 2004

Jagow/Steger (2005): Bettina von Jagow, Florian Steger (Hrsg.), Literatur und Medizin. Ein Lexikon, Göttingen 2005

Jagow/Steger (2006): Bettina von Jagow, Florian Steger (Hrsg.), Jahrbuch Literatur und Medizin, Heidelberg 2006

Kesey (1962): Ken Kesey, One flew over the cuckoo's nest, New York 1962

Kesey (1996): Ken Kesey, Einer flog über das Kuckucksnest, Hamburg 1996

Koch (1976): Egmont R. Koch, Chirurgie der Seele. Operative Umpolung des Verhaltens, Stuttgart 1976

Maio (2004): Giovanni Maio, Die Geschichte der psychiatrischen Klinik im Spiegel des Films, in: Axel Dost, Gudrun Färber-Töller, Heinz Rodegra (Hrsg.), Hospital - Kunst - Medizin, Aachen 2004, S. 153-182

Moniz (1949): António Caetano de Abreu Freire Egas Moniz, Die präfrontale Leukotomie, Archiv für Psychiatrie 181 (1949), S. 591-602

Müller (2007): Sabine Müller, Dilemmata bei operativen Eingriffen in das Gehirn, in: Dominik Groß, Sabine Müller (Hrsg.), Sind die Gedanken frei? Die Neurowissenschaften in Geschichte und Gegenwart, Berlin 2007, S. 175-207

Müller-Seidel (1997): Walter Müller-Seidel, Arztbilder im Wandel. Zum literarischen Werk Arthur Schnitzlers, München 1997

News (1949): News and Views. Nobel Prize for Physiology and Medicine for 1949, Nature 4179 (1949), p. 947

Röckerath (2004): Klaus Röckerath, Stereotaxie. Kognitive Therapie ist nicht alleiniger Maßstab, Deutsches Ärzteblatt 101 (2004), S. A-3021/B-2556/C-2435

Schipperges (1955): Heinrich Schipperges, Zur Entwicklung der Psychochirurgie, Ciba-Zeitschrift 75 (1955), S. 2491-2497

Schnitzler (1988): Arthur Schnitzler, Medizinische Schriften, hrsg. von Horst Thomé, Wien 1988

Sigusch (1977): Volkmar Sigusch, Medizinische Experimente am Menschen. Das Beispiel Psychochirurgie, Berlin 1977 (= Jahrbuch für kritische Medizin, 2)

Sontag (1977): Susan Sontag, Illness as metaphor, New York 1977

Sontag (1988): Susan Sontag, Aids and Its Metaphors, New York 1988

Steger (2003): Florian Steger, Medien, Sucht und Kultur. Das Potential medialer Repräsentationen von Sucht für das Verständnis psychopathologischer Phänomene, Fundamenta Psychiatrica 17 (2003), S. 53-57

Thomé (1993): Horst Thomé, Autonomes Ich und „Inneres Ausland“. Studien über Realismus, Tiefenpsychologie und Psychiatrie in deutschen Erzähltexten (1848-1914), Tübingen 1993

Voderholzer (2004): Ulrich Voderholzer, Stereotaxie. Tabu gebrochen, Deutsches Ärzteblatt 101 (2004), S. A$3020 / B-2551 / C-2434$ 


\section{E Zwangsbehandlung}

\section{Internet}

www.alterpsy.org/de/chirurgie.php

www.djfl.de/entertainment/stars/j/jane_campion.html.

www.doctissimo.fr/html/psychologie/mag_2002/mag0614/ps_5618_psychochirurgie.htm www.imdb.com/name/nm0001719/

www.imdb.com/title/tt0072267/

www.imdb.com/title/tt009g040/

http://jeffreykauffman.net/francesfarmer/sheddinglight.html

www.litencyc.com/php/speople.php?rec=true\&UID $=4941$

www.sensesofcinema.com/contents/directors/02/campion.html

http://de.wikipedia.org/wiki/Einer_flog_\%C3\%BCber_das_Kuckucksnest

http://de.wikipedia.org/wiki/Frances_Farmer

http://de.wikipedia.org/wiki/Ken_Kesey

http://en.wikipedia.org/wiki/Ken_Kesey 